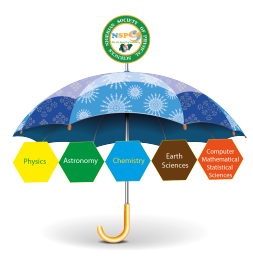

\title{
First-principles calculations of Fluorine-doped Titanium dioxide: A prospective material for solar cells application
}

\author{
A. Shamsudeen ${ }^{\text {b }}$, A. Shuaibu ${ }^{\mathrm{a}, *}$, S. G. Abdu ${ }^{\mathrm{a}}$, M. S. Abubakar ${ }^{\mathrm{a}}$, Abdullahi lawal $^{\mathrm{b}}$ \\ ${ }^{a}$ Department of Physics, Kaduna State University, P. M. B. 2339, Kaduna, Nigeria \\ ${ }^{b}$ Department of Physics, Federal College of Education Zaria, Nigeria
}

\begin{abstract}
This study focuses on the anatase $\mathrm{TiO}_{2}$ doped Fluorine to investigate their structural and electronics properties using Density Functional Theory (DFT) within generalized gradient approximation (GGA) as implemented in Quantum ESPRESSO (QE). For the anatase $\mathrm{TiO}_{2}$ phase the calculated electronic band structures of pure $\mathrm{TiO}_{2}$ and $\mathrm{TiO}_{2}$ doped Fluorine nanocrystals are displayed along a high symmetry directions and the energy range of band structure is plotted from $0.0 \mathrm{eV}$ to $3.9 \mathrm{eV}$, the energy separation between the bottom of the conduction band and the top of valence band occurred at the $\Gamma$ and $\mathrm{N}$ points, indicating that anatase $\mathrm{TiO}_{2}$ is an indirect band gap material with an approximate value of $2.30 \mathrm{eV}$ energy gap, this value is consistent with previous DFT result. When $F$ is added the band structure did not change much because fluorine element doping is conducive to the generation of Oxygen holes and enhances the mobility of effective electrons which can enhance the conductivity of the adsorbent substrate and improve the solar cell performance of the fluorine-doped $\mathrm{TiO}_{2}$. The band gap value obtained for $F$ doped $\mathrm{TiO}_{2}$ was found to be $2.11 \mathrm{eV}$. The dopant formation energy of Fluorine is calculated to be $-55.6 \mathrm{Ry}$ which is equivalent to $-756.5 \mathrm{eV}$.
\end{abstract}

Keywords: DFT, $\mathrm{TiO}_{2}$, Fluorine, electronic properties, solar cells

Article History :

Received: 26 August 2019

Received in revised form: 10 October 2019

Accepted for publication: 11 October 2019

Published: 17 December 2019

(C)2019 Journal of the Nigerian Society of Physical Sciences. All rights reserved. Communicated by: B. J. Falaye

\section{Introduction}

Engineers and scientists show so much interest in compounds made of transition metal oxides which have wide band gap, very good light absorption capability, very good value of heat formation and other various fascinating properties. This is because these properties present the potential of use in advanced applications such as solar cells $[1,2,3]$. Nowadays a lot of investigations are carried out on oxide materials, especially to meet industry requirements. This is because material oxides are used in a lot of art technologies such as magnetic disks, ICs and solar cells and their likes. A lot of properties sought

${ }^{*}$ Corresponding author tel. no: +2348179931945

Email address: alhazikara@gmail.com (A. Shuaibu ) by researchers and the industry, which is driving more research into the area, include need for economical alternative to current materials, non-toxic and environmentally friendly, wide availability and good electrical, magnetic and or optical properties for use as base materials or doping in optical fibers and similar uses. These demands are partly driven by need to meet energy demand in the future $[3,4,5]$. One of the oxides which attracted a lot of theoretical and experimental research effort is $\mathrm{TiO}_{2}$ [6]. This is in order to better understand the optoelectronic properties of $\mathrm{TiO}_{2}$. Many naturally occurring and engineered polymorphs of $\mathrm{TiO}_{2}$ exist. These include anatase, baddeleyite, pyrite, columbite, brookite, fluorite, cotunnite and rutile. Three of these (i.e. anatase, brookite and rutile) exist naturally while the rest are manufactured. Due to popular demand and remark- 
able properties of the naturally occurring polymorphs such as rutile, for use in production of catalyst, flat panel displays, optoelectronic devices, sensors, and solar cell technology and so on, many of them are often artificially synthesized.

Rutile, as a result of its structure, possesses one of the highest stabilities that can be found as compared to others. Anatase is also thermodynamically stable up to a temperature of about $800{ }^{\circ} \mathrm{C}$. On another hand brookite has a structure that resembles that of rutile, though with less stability at high temperatures $[7,8]$. The structure of anatase is the most suitable for photo catalytic applications. It is one of the reasons for attracting a lot of research interest into the properties of $\mathrm{TiO}_{2}$. Other properties of $\mathrm{TiO}_{2}$, for which it is held in high regards is its use in many ceramic materials to add features and capabilities such as being hydrophilic, photo catalytic, hydrophobic and having antibacterial features [9], this is aside its good chemical stability, nontoxicity and reduced cost. $\mathrm{TiO}_{2}$ is also used for air and water purification by taking advantage of its antibacterial capabilities. It is used in PVC fabrics, used to make materials with self-cleaning effects, used for preservation of glass and cultural heritage [10]. It is also used in de-synthesizing photo cells as a common photocathode.

The history of $\mathrm{TiO}_{2}$ went as far back as 1971 when the work of Fushijima and Honda revealed the photo electrochemistry of the material while using it as an anode in an electrochemical cell. This application is what gave rise to all the interest that was generated over the years which led to discovery of a lot of its other properties [11]. Wang et al.[12] reported that $\mathrm{TiO}_{2}$, when used to fabricate ultra-violet (UV) photo-detector, shows more energy efficient operation than other material such as Gallium Nitride $(\mathrm{GaN})$, Silicon $(\mathrm{S} i)$ and Zinc Oxide $(\mathrm{ZnO})$. Its wide band gap is what limits the usage of $\mathrm{TiO}_{2}$ as a semiconductor directly used for semiconductor component. This is why it is mostly used as a substrate for other semiconductor materials especially in a thin film solar cell technology.

Because of these multiple uses and benefits that can be derived from $\mathrm{TiO}_{2}$, it is obviously of paramount importance, to areas of harnessing solar energy, optoelectronics, capturing visible and near visible light, to unveil the full potentials of this compound and its various forms. This should be done through tuning the properties of the oxides such as its band gap and so on. This need is the drive for theoretical and experimental researches in order to have the comprehensive knowledge regarding its structure, properties and their relationship to its performance. A lot of work, especially experimental work is now available in the research community regarding $\mathrm{TiO}_{2}$, but theoretical results of research related to the compilation that are adequate to characterize it are still scarce. Currently most of the theoretical works are at the level of investigations which were performed via Density Function Theory (DFT). Since it gives a simple basis to characterized the quantum properties of various materials and plays an important role in designing many new materials. In addition to many experimental studies of $\mathrm{TiO}_{2}$, is seen by many as a favorable material for many important high technological applications, a few important first principle DFT studies are reported in previous work. Furthermore, the studies discussed and many others, the band gap values of the materials are either over- or under-estimated. This results in deducting contradictory nature of the band gaps which highlights the need for more studies. In addition, a lot of the work was done at different side by side of GGA and LDA, a technique which underestimate the band gap. Thus, to better understand the optoelectronic properties of $\mathrm{TiO}_{2}$ such as strong light absorption, photocurrent sensitivity to the polarization of light it is essential to use reliable XC approximations to determine its electronic properties. To utilize the maximum spectral range in the solar spectrum, the band gap of $\mathrm{TiO}_{2}$ should be tuned, which will broaden the operational optical window of the $\mathrm{TiO}_{2}$ -based optoelectronic devices.

Nowadays, doping is one of the well-approaches to improve the properties of materials. Doping can dramatically modify physical and chemical properties of materials [13]. Therefore, $\mathrm{TiO}_{2}$ is repeatedly doped with different metals and transition elements to tune its band gap and enhance its optical, electrical, and magnetic properties [14, 15, 16, 17, 18]. Non-metal doping is another approach used to narrow the band gap; in comparison to metal doping that often forms a donor level in the forbidden band, non-metal doping usually shifts the valence band edge upward [18]. Doping with non-metal elements such as $F$ shows an enhancement in the electronic, optical, and magnetic properties of narrow-band gap semiconductor materials [19, 20, 21]. The doping by $\mathrm{F}$ has also shown higher thermal stability and conductivity in graphene [22]. The aforesaid reasons motivated us to study the doping by Fluorine atom, which can significantly modify/tune the electronic properties $\mathrm{TiO}_{2}$. To the best of our knowledge, no theoretical studies on F-doped $\mathrm{TiO}_{2}$ exist in literature. The Fluorine doping approach in $\mathrm{TiO}_{2}$ may open new paths to non-metal elements doping for various other potential applications such as infrared detectors, infrared LEDs, lasers, transistors, and thermo-photovoltaic systems.

\section{COMPUTATIONAL DETAILS}

\subsection{Computational method}

The calculations are performed on the $2 \times 2 \times 2$ supercells relative to the standard primitive unit cell of Fluorine doped anatase $\mathrm{TiO}_{2}$ within first principle calculation using Quantum ESPRESSO simulation package [23]. Perdew-Burke-Ernzerhof generalized gradient approximation (PBE-GGA) exchange-correlation potential [24] were used for treating electron-electron effects. For integrals, smearing has been adopted and to be specific Maxfessel-Paxton smearing method. The brillouin zone integration is performed using Monkhorst-Pack scheme [25] with $3 \times 3 \times 2 \mathrm{k}$-points grids for all the materials. For fluorine doped $\mathrm{TiO}_{2}$ structure $2 \times 2 \times 2$ supercells relative to the standard conventional unit cell were used. The supercell consists of twelve numbers of atoms: four Titanium atom and eight Oxygen atoms. One $O$ atom was replaced by Fluorine atom making $0.25 \%$ occupancy by the dopant. The super cell dimensions are kept fixed throughout the calculations, while the atomic positions are fully relaxed for all calculations using BroydenFletcher-Golfarb-Shannon (BFGS) algorithm, until the forces acting on the atoms are below $0.001 \mathrm{eV} / \AA$. 


\section{RESULTS AND DISCUSSION}

\subsection{Convergence Test}

In any DFT calculation using DFT code, it is of paramount importance to perform a convergence test calculation before commencing the actual calculation. The results presented below represent the convergence test with respect to plane wave kinetic energy cut-off and k-points mesh for undoped anatase $\mathrm{TiO}_{2}$ and Fluorine atom doped $\mathrm{TiO}_{2}$.

\subsubsection{Convergence Test Results of undoped $\mathrm{TiO}_{2}$}
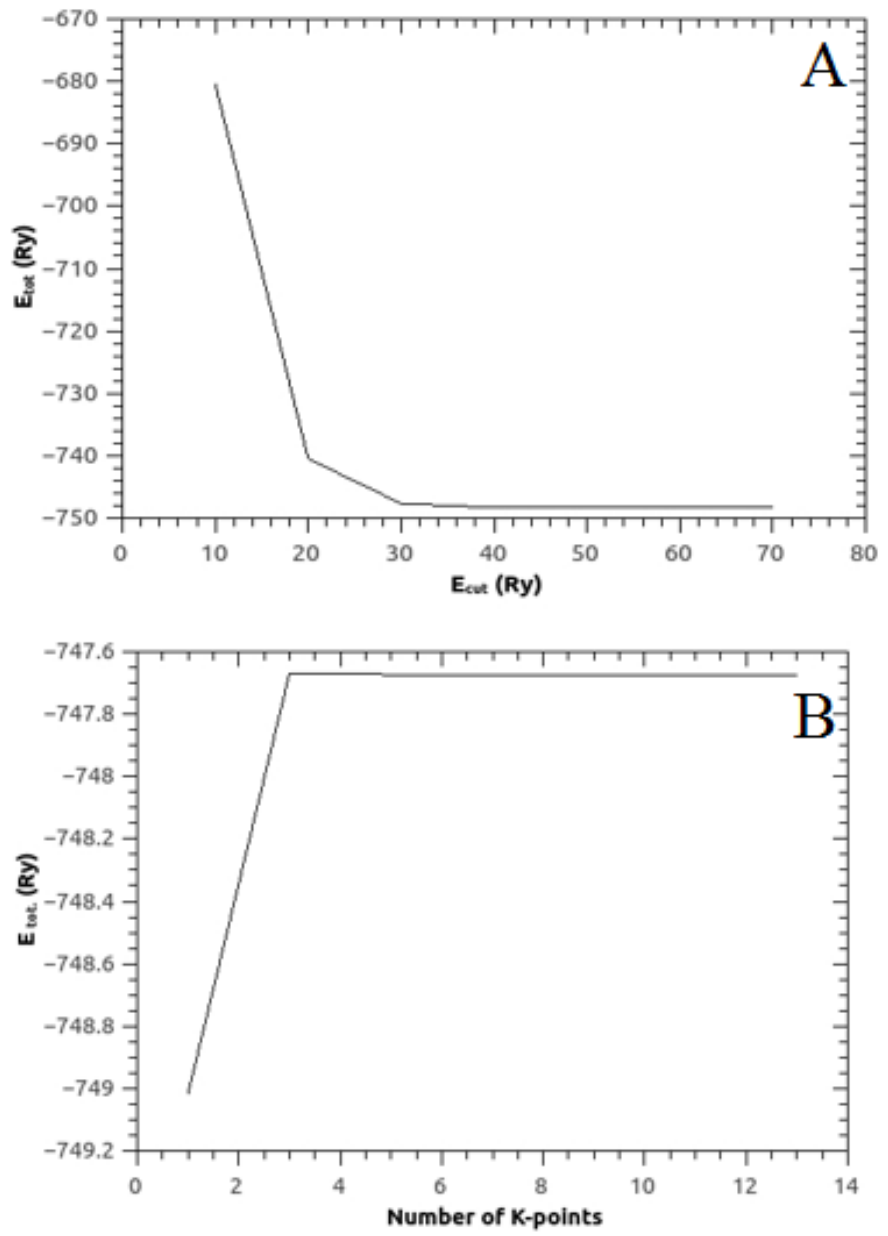

Figure 1: (A) the convergence of total energy with respect to the kinetic energy cut-off. (B) The convergence of the total energy with respect to the k-points grids.

\subsubsection{Convergence Test Results of doped Fluorine atom.}

It could be seen from Figure 1 that the total energy changes considerably with the kinetic energy cut-off, until at some energy cut-off where it becomes almost stable. In all the two cases, as the total energy decreases, the kinetic energy cut-off increases from $10 R y$ to $40 R y$ and becomes almost stable at $40 R y$. That is to say, the total energy remains constant with any further increases in the kinetic energy cut-off from $40 R y$. This indicates a well-converged energy cut-off. That is why;
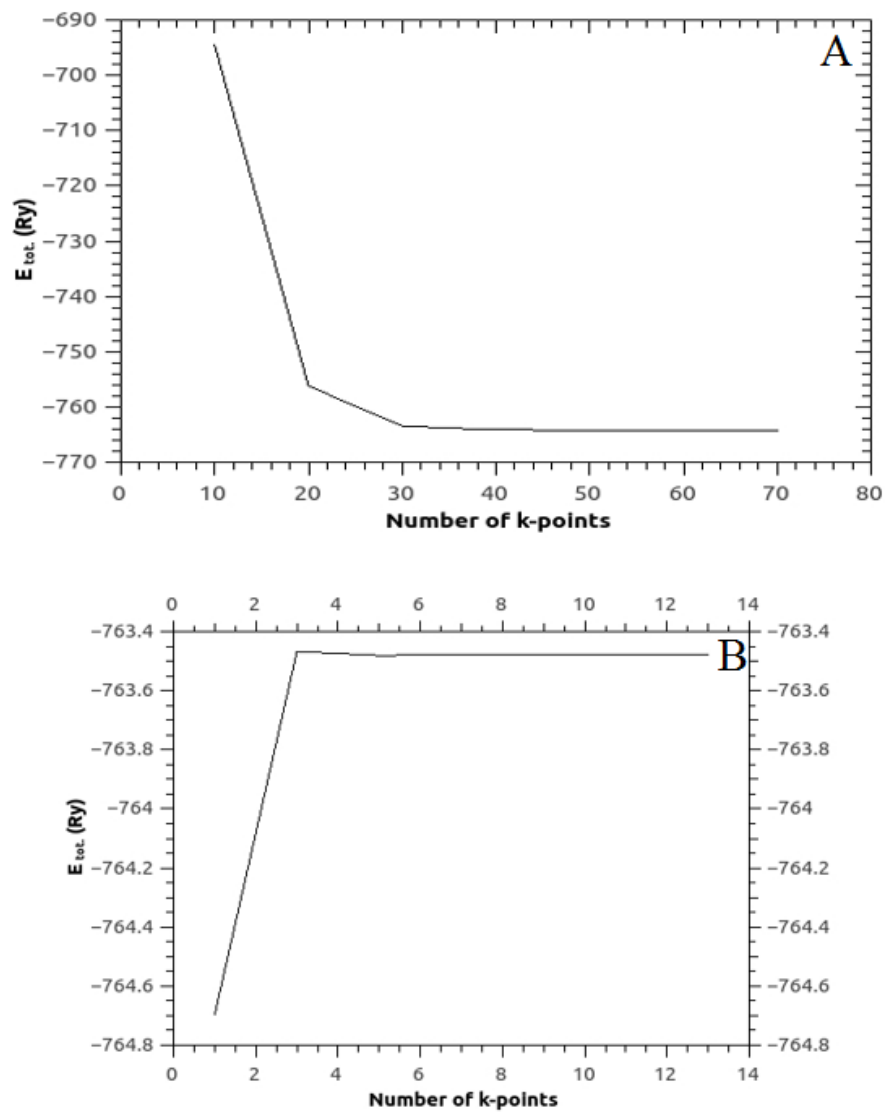

Figure 2: (A) NO HEADING. (B) NO HEADING.

$40 R y$ was used as the plane wave basis set for the kinetic energy cut-off of the two cases.

However, Figure 2B and Figure 5B show the variations of the total energy with respect to the k-points grids. The total energy changes considerably with the number of k-points at a certain points, showing a well-converged value. In all the two cases, the total energy increases from $1 \times 1 \times 2$ to $3 \times 3 \times 2 \mathrm{k}$-point grids and become almost stable at $3 \times 3 \times 2$. As such, $3 \times 3 \times 2$ k-points have been adopted for the three cases. Furthermore, Monkhorst and Pack method of selecting k-points is commonly used in most DFT calculations [25]. Most of the DFT codes provide ways of choosing k-points using Monkhorst and Pack method.

\subsection{Structural Properties of the Undoped $\mathrm{TiO}_{2}$ and $\mathrm{TiO}_{2}$ doped $F$.}

In order to explore the structural properties of the anatase $\mathrm{TiO}_{2}$ with space group 14/amd was simulated in Figure 3. The anatase structure exhibit tetragonal geometry and have a high symmetry in which six Oxygen atoms is surrounded to each $T i$ atom.

From Table 5, it can be observed that, for $12.5 \%$ substitutional doping case, there are eight symmetrically different approach in which Oxygen $(O)$ atom can be replaced by Fluorine $(F)$ atom(s). But for this particular study, as shown in tables above, one configuration $\left(D_{8_{-}} 0.125\right)$ is considered. No structural transition is seen; as such the crystal parameters remained 
Table 1: Convergence test of $\mathrm{TiO}_{2}$ total energy with respect to kinetic energy cut-off of the plane wave of undoped $\mathrm{TiO}_{2}$.

\begin{tabular}{lcc}
\hline S/N & Kinetic Energy Cut-Off (Ry) & Total Energy (Ry) \\
\hline 1 & 10 & -680.917 \\
2 & 20 & -740.466 \\
3 & 30 & -747.717 \\
4 & 40 & -747.374 \\
5 & 50 & -748.413 \\
6 & 60 & -748.421 \\
7 & 70 & -748.429 \\
\hline
\end{tabular}

Table 2: Convergence of the total energy with respect to the k-points grids of undoped $\mathrm{TiO}_{2}$.

\begin{tabular}{lcc}
\hline S/N & NUMBER OF K-POINTS & Total Energy $(\mathrm{Ry})$ \\
\hline 1 & $1 \times 1 \times 2$ & -749.018 \\
2 & $3 \times 3 \times 2$ & -747.670 \\
3 & $5 \times 5 \times 2$ & -747.673 \\
4 & $7 \times 7 \times 2$ & -747.673 \\
5 & $9 \times 9 \times 2$ & -747.672 \\
6 & $11 \times 11 \times 2$ & -747.673 \\
7 & $13 \times 13 \times 2$ & -747.674 \\
\hline
\end{tabular}

Table 3: Convergence test of total energy with respect to the kinetic energy cut-off.

\begin{tabular}{lcc}
\hline S/N & KINETIC ENERGY CUT-OFF (Ry) & TOTAL ENERGY (Ry) \\
\hline 1 & 10 & -694.517 \\
2 & 20 & -756.204 \\
3 & 30 & -763.468 \\
4 & 40 & -764.135 \\
5 & 50 & -764.174 \\
6 & 60 & -764.183 \\
7 & 70 & -764.191 \\
\hline
\end{tabular}

Table 4: Convergence of the total energy with respect to the k-points grids of undoped $\mathrm{TiO}_{2}$.

\begin{tabular}{lcc}
\hline S/N & NUMBER OF K-POINTS & TOTAL ENERGY $(\mathrm{Ry})$ \\
\hline 1 & $1 \times 1 \times 2$ & -764.697 \\
2 & $3 \times 3 \times 2$ & -763.468 \\
3 & $5 \times 5 \times 2$ & -763.481 \\
4 & $7 \times 7 \times 2$ & -763.478 \\
5 & $9 \times 9 \times 2$ & -763.478 \\
6 & $11 \times 11 \times 2$ & -763.479 \\
7 & $13 \times 13 \times 2$ & -763.480 \\
\hline
\end{tabular}

Table 5: Configurations for substitutional doping of $\mathrm{O}$ by $\mathrm{F}$ anatase $\mathrm{TiO}_{2}$ material.

\begin{tabular}{lllllllll}
\hline Undoped & $O$ & $O$ & $O$ & $O$ & $O$ & $O$ & $O$ & $O$ \\
\hline$D_{1 \_} 0.125$ & $F$ & $O$ & $O$ & $O$ & $O$ & $O$ & $O$ & $O$ \\
\hline$D_{2 \_} 0.125$ & $O$ & $F$ & $O$ & $O$ & $O$ & $O$ & $O$ & $O$ \\
\hline$D_{3 \_} 0.125$ & $O$ & $O$ & $F$ & $O$ & $O$ & $O$ & $O$ & $O$ \\
\hline$D_{4_{-}} 0.125$ & $O$ & $O$ & $O$ & $F$ & $O$ & $O$ & $O$ & $O$ \\
\hline$D_{5} 0.125$ & $O$ & $O$ & $O$ & $O$ & $F$ & $O$ & $O$ & $O$ \\
\hline$D_{b_{-}} 0.125$ & $O$ & $O$ & $O$ & $O$ & $O$ & $F$ & $O$ & $O$ \\
\hline$D_{7 \_} 0.125$ & $O$ & $O$ & $O$ & $O$ & $O$ & $O$ & $F$ & $O$ \\
\hline$D_{8_{-}} 0.125$ & $O$ & $O$ & $O$ & $O$ & $O$ & $O$ & $O$ & $F$ \\
\hline
\end{tabular}



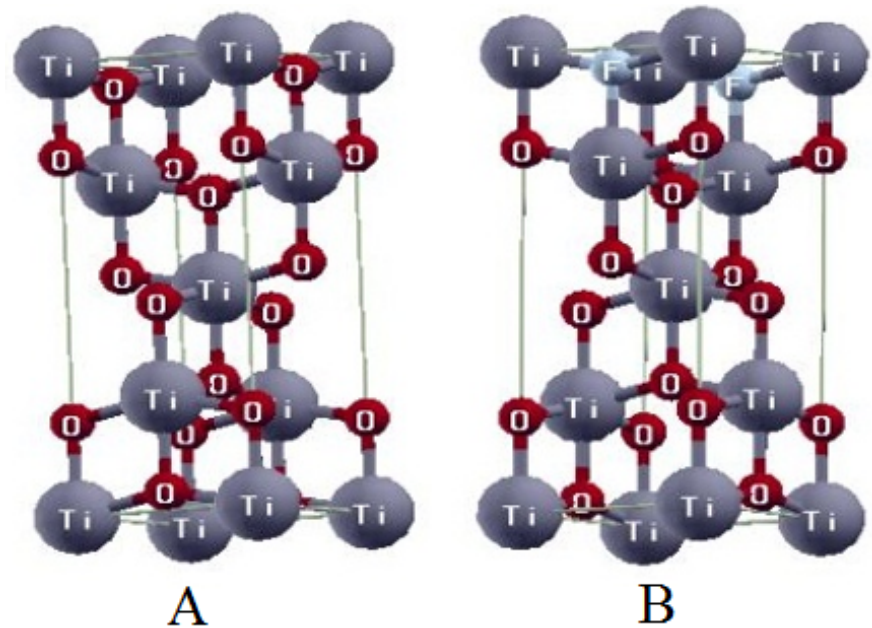

Figure 3: (A): Anatase Structure for undoped $\mathrm{TiO}_{2}$ and (B) $\mathrm{TiO}_{2}$ doped F.

the same as that for the undoped system. To find the stability of the structure after Fluorine doping, the dopant formation energy of the Fluorine atom is estimated using Equation 1 [26]. The dopant formation energy in this context simply refers to the energy needed to insert one Fluorine atom with a chemical potential $\mu_{F}$ into the supercell after removing one Oxygen atom with chemical potential $\mu_{F}$ from the same position [27].

$$
E_{f}=E_{\text {doped }}-E_{\text {undoped }}+\mu_{o}-\mu_{F}
$$

where, $E_{\text {doped }}$ is the total energy of the anatase $\mathrm{TiO}_{2}$ material; $E_{\text {undoped }}$ is the total energy of the anatase undoped $\mathrm{TiO}_{2}$ system; $\mu_{o}$ is the chemical potential per atom of Oxygen bulk crystal; $\mu_{F}$ is the chemical potential per atom of Fluorine bulk crystal.

Following common practice, the chemical potentials were found as the DFT total energy per atom in the bulk systems. The dopant formation energy of Fluorine is calculated to be $-55.6 R y$ which is equivalent to $-756.5 \mathrm{eV}$. This serves as the measure of the stability of the doped structure, the lower value of the formation energy signifies the most stable structure. From this value of dopant formation energy, this shows that, the Fluorine doped $\mathrm{TiO}_{2}$ is stable.

\subsection{Electronic Properties}

Electronic properties calculations are very crucial for describing the optoelectronic properties of solids. The electronic properties investigations of $\mathrm{TiO}_{2}$, doped Fluorine covers the electronic band structure, density of state (DOS) and partial density of state (PDOS). The main purpose of the ground state, electronic band structure, DOS and PDOS calculations in this work is to obtain KS eigenvalues and eigenfunctions as well as useful information about the electronic properties of the concerned materials. To understand the effect of doping on $\mathrm{TiO}_{2}$, Fluorine $(F)$ was added in the calculations. The calculated electronic band structures of pure $\mathrm{TiO}_{2}$ and $\mathrm{TiO}_{2}$ doped Fluorine thin film are displayed along the eight symmetry $(\Gamma \rightarrow H \rightarrow$ $N \rightarrow P \rightarrow \Gamma \rightarrow X \rightarrow M \rightarrow R$ ) directions and the energy range of band structure is plotted from $0.0 \mathrm{eV}$ to $3.9 \mathrm{eV}$. The Fermi level position on the band structure of these crystals is shown by the zero on the energy scale. PBE exchange correlation potentials is chosen over LDA, because in several cases GGA-PBE gives more reliable and accurate results for DFT electronic calculation. The energy separation between the bottom of the conduction band and the top of valence band occurred at the $\Gamma$ and $N$ points or band structure calculations within PBE which indicate that anatase $\mathrm{TiO}_{2}$ is an indirect band gap material with value of $2.30 \mathrm{eV}$ energy gap, this value is consistent with previous DFT result. However, the value is smaller than experimental result of $3.21 \mathrm{eV} \mathrm{[28]} \mathrm{and} \mathrm{this} \mathrm{effect} \mathrm{is} \mathrm{the} \mathrm{limitation}$ of DFT approach due to approximations used in the exchangecorrelation functional. On the other hand, the $\mathrm{F}$-doped $\mathrm{TiO}_{2}$ model was established based on the perfect crystal plane model. A fluorine atom replaces one of the $O$ atoms on the surface of the anatase $\mathrm{TiO}_{2}$ perfect crystal plane, and $\mathrm{F}$ atom combined with $T i$ atoms to form $T i-F$ bonds. It can be seen that the energy band gap did not change much after the fluorine atom being doped and the value obtained was found to be $2.11 \mathrm{eV}$. Furthermore, fluorine element doping is conducive to the generation of Oxygen holes and enhances the mobility of effective electrons, which can enhance the conductivity of the adsorbent substrate and improve the solar cell performance of the fluorinedoped $\mathrm{TiO}_{2}$.

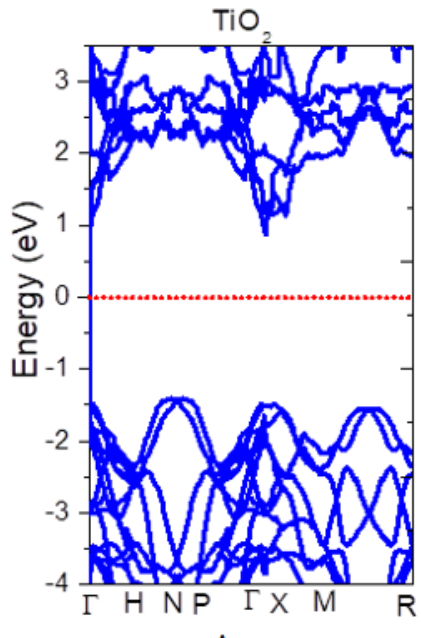

A

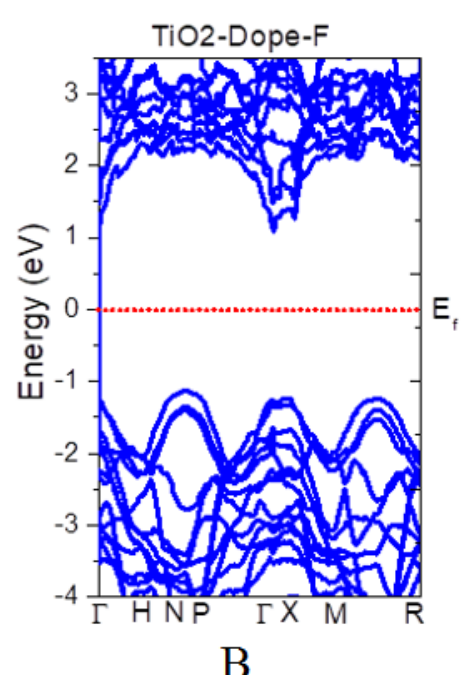

$\mathrm{B}$
Figure 4: Band structure of (A) Pure $\mathrm{TiO}_{2}$ (B) $\mathrm{F}$ doped $\mathrm{TiO}_{2}$

For more clarification for the nature of the energy gap, we have also study the total density of state (DOS) of anatase $\mathrm{TiO}_{2}$, and $\mathrm{F}$ doped anatase $\mathrm{TiO}_{2}$. Figure 5 shows a decomposition of the calculated total DOS of $\mathrm{TiO}_{2}$ and $\mathrm{F}$ doped $\mathrm{TiO}_{2}$ in bulk forms. For pure $\mathrm{TiO}_{2}$ the lowest valence states is dominated by $T i$-s orbital and $O$-p orbital while in the cased of $F$ doped $\mathrm{TiO}_{2}$ the lowest valence states is dominated by $T i$-s orbital, $O-\mathrm{p}$ orbital and $F$-p orbital. The $T i$-d orbital and $O$-s orbital contribute much high in the conduction band for pure $\mathrm{TiO}_{2}$ while $T i$-d, $O$-s, $O$-s orbital as well as $F$-s orbital contributed slightly higher in the conduction band. The $T i$-p and $O$-p orbitals of $\mathrm{TiO}_{2}$ contribute a slightly higher in the valence band near the Fermi level. For $\mathrm{F}$ anatase doped $\mathrm{TiO}_{2}$ the main contribution 
Table 6: Calculated energy gap of $\mathrm{TiO}_{2}$ and $\mathrm{TiO}_{2}$ doped $\mathrm{F}$ with previous first principle calculations and experimental data.

\begin{tabular}{lcccc}
\hline Material & Work & Method & Band gap $E_{g}(\mathrm{eV})$ & Nature \\
\hline Pure $\mathrm{TiO}_{2}$ & Our Work & PBE-GGA & 2.30 & Indirect \\
& Other Work & PBE-GGA[29] & 2.14 & Indirect \\
& & EV-GGA[29] & 2.32 & Indirect \\
& & PBE-GGA[30] & 2.30 & Indirect \\
& & PBE-GGA[31] & 2.30 & Indirect \\
& & Experiment[32] & 3.21 & Indirect \\
$\mathrm{F}^{\text {doped } \mathrm{TiO}_{2}}$ & Our Work & PBE-GGA & 2.11 & Indirect \\
\hline
\end{tabular}

of valence band near the Fermi level is from $T i$-p orbital and $O$ p orbital. The $T i$-s, $O$-s and $O$-p orbitals contributed the most near the Fermi level, thus held responsible for the properties of $\mathrm{TiO}_{2}$. Also, s- and p-orbitals of $\mathrm{Ti}$ atoms, s- and p-orbitals of $\mathrm{O}$ atoms and s-orbital of $\mathrm{F}$ atoms of $\mathrm{F}$ doped $\mathrm{TiO}_{2}$ compound contributed the most near the Fermi level, thus held responsible for the properties.

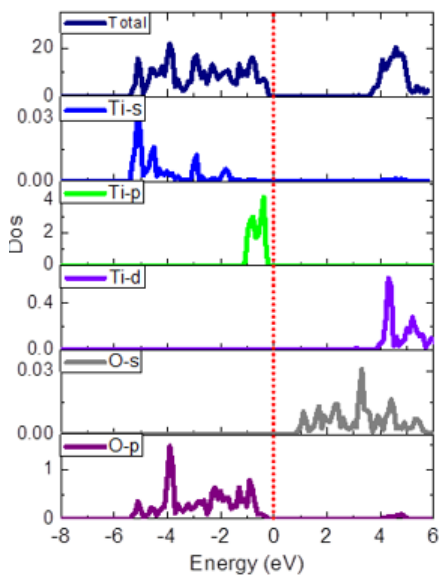

A

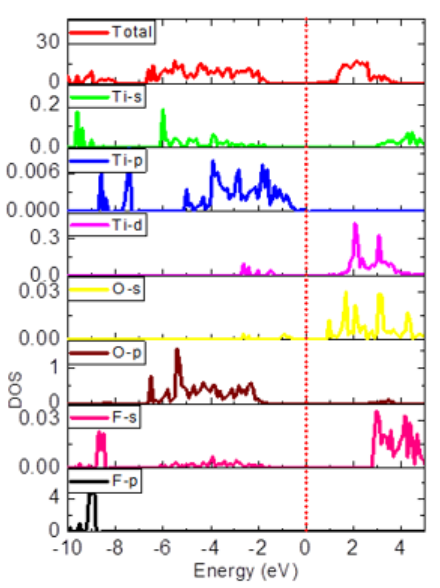

B
Figure 5: Total and partial density of state (A) Pure $\mathrm{TiO}_{2}$ (B) $\mathrm{F}$ doped $\mathrm{TiO}_{2}$.

\section{Conclusion}

A first principle study was used using PBE XC functional in Quantum ESPRESSO code. The available results were compared with other experimental values. The electronic properties density of state, partial density of state and band gap values were calculated in the ground state properties. It was found that $\mathrm{TiO}_{2}$ in anatase phase has indirect band gap with a value of $2.30 \mathrm{eV}$, this value is consistent with previous DFT results. The obtained band gap value is smaller than experimental result of $3.21 \mathrm{eV}$ and this effect is the limitation of DFT approach due to approximations used in the exchange-correlation functional. The Fluorine doped $\mathrm{TiO}_{2}$ model was established based on the perfect crystal plane model. A fluorine atom replaces one of the $O$ atoms on the surface of the anatase $\mathrm{TiO}_{2}$ perfect crystal plane, and $F$ atom combined with $T i$ atoms to form $T i-F$ bonds. It can be seen that the energy band gap did not change much after the fluorine atom being doped. Furthermore, fluorine element doping is conducive to the generation of Oxygen holes and enhances the mobility of effective electrons which can enhance the conductivity of the adsorbent substrate and improve the solar cell performance of the fluorine-doped $\mathrm{TiO}_{2}$. The dopant formation energy of Fluorine is calculated to be $-55.6 R$ which is equivalent to $-756.5 \mathrm{eV}$. The formation energy serves as the measure of the stability of the doped structure; the lower value of the formation energy signifies the most stable structures [26]. From this value of dopant formation energy, this shows that the Fluorine doped anatase $\mathrm{TiO}_{2}$ is stable.

\section{Acknowledgments}

We thank the referees for the positive enlightening comments and suggestions, which have greatly helped us in making improvements to this paper.

\section{References}

[1] E. Kabir, P. Kumar, S. Kumar, A. A. Adelodun \& K.-H. Kim, " Solar energy: Potential and future prospects"', Renewable and Sustainable Energy Reviews 82 (2018) 894.

[2] J. Robertson \& B. Falabretti, " Electronic structure of transparent conducting oxides, Handbook of transparent conductors", Springer (2011) pp27-50.

[3] K. Nagaveni, M. Hegde, N. Ravishankar, G. Subbanna \& G. Madras, "Synthesis and structure of nanocrystalline $\mathrm{TiO}_{2}$ with lower band gap showing high photocatalytic activity", Langmuir 20 (2004) 2900.

[4] Y. Xiao, C. Wang, K. K. Kondamareddy, P. Liu, F. Qi, H. Zhang, S. Guo \& X.-Z. Zhao," 'Enhancing the performance of hole-conductor free carbonbased perovskite solar cells through rutile-phase passivation of anatase $\mathrm{TiO}_{2}$ scaffold", Journal of Power Sources 422 (2019) 138.

[5] J. Y. Seo, R. Uchida, H. S. Kim, Y. Saygili, J. Luo, C. Moore, J. Kerrod, A. Wagstaff, M. Eklund \& R. McIntyre, "Boosting the Efficiency of Perovskite Solar Cells with CsBr-Modified Mesoporous $\mathrm{TiO}_{2}$ Beads as Electron-Selective Contact", Advanced Functional Materials 28 (2018) 1705763.

[6] T. Singh, S. Öz, A. Sasinska, R. Frohnhoven, S. Mathur \& T. Miyasaka, "Sulfate-Assisted Interfacial Engineering for High Yield and Efficiency of Triple Cation Perovskite Solar Cells with Alkali-Doped $\mathrm{TiO}_{2}$ Electron-Transporting Layers", Advanced Functional Materials 28 (2018) 1706287.

[7] A. Parameswari, Y. Soujanya \& G. N. Sastry, "'Functionalized Rutile $\mathrm{TiO}_{2}$ (110) as a Sorbent To Capture $\mathrm{CO}_{2}$ through Noncovalent Interactions: A Computational Investigation", The Journal of Physical Chemistry C 123 (2019) 3491.

[8] Y. Wang, A. S. Ganeshraja, C. Jin, K. Zhu \& J. Wang, "One-pot synthesis visible-light-active $\mathrm{TiO}_{2}$ photocatalysts at low temperature by peroxotitanium complex", Journal of Alloys and Compounds 765 (2018) 551-559. 
[9] O. Çomaklı, M. Yazıcı, H. Kovacı, T. Yetim, A. Yetim \& A. Çelik, "Tribological and electrochemical properties of $\mathrm{TiO}_{2}$ films produced on $\mathrm{Cp}-\mathrm{Ti}$ by sol-gel and SILAR in bio-simulated environment", Surface and Coatings Technology 352 (2018) 513.

[10] M. Gurbuz, B. Atay \& A. Dogan, "Synthesis of High-Temperature-Stable $\mathrm{TiO}_{2}$ and its Application on Ag+-Activated Ceramic Tile", International Journal of Applied Ceramic Technology 12 (2015) 426.

[11] H. Gao, X. Li, J. Lv \& G. Liu, "Interfacial charge transfer and enhanced photocatalytic mechanisms for the hybrid graphene/anatase $\mathrm{TiO}_{2}$ (001) nanocomposites", The Journal of Physical Chemistry C 117 (2013) 16022-16027.

[12] W. Wang, C. Shan, H. Zhu, F. Ma, D. Shen, X. Fan \& K. Choy, "Metalinsulator-semiconductor-insulatormetal structured titanium dioxide ultraviolet photodetector", Journal of Physics D: Applied Physics 43 (2010) 045102.

[13] A. G. Ilie, M. Scarisoreanu, E. Dutu, F. Dumitrache, A. -M. Banici, C. T. Fleaca, E. Vasile \& I. Mihailescu, "Study of phase development and thermal stability in as synthesized $\mathrm{TiO}_{2}$ nanoparticles by laser pyrolysis: ethylene uptake and oxygen enrichment"', Applied Surface Science $\mathbf{4 2 7}$ (2018) 798

[14] P. Giannozzi, S. Baroni, N. Bonini, M. Calandra, R. Car, C. Cavazzoni, D. Ceresoli, G. L. Chiarotti, M. Cococcioni \& I. Dabo, "QUANTUM ESPRESSO: a modular and open-source software project for quantum simulations of materials", Journal of physics: Condensed matter 21 (2009) 395502.

[15] J. P. Perdew, K. Burke \& M. Ernzerhof, "'Generalized gradient approximation made simple", Physical review letters 77 (1996) 3865.

[16] H. J. Monkhorst \& J. D. Pack, "Special points for Brillouin-zone integrations", Physical Review B 13 (1976) 5188.

[17] X. Fan, F. Wang, Z. Chu, L. Chen, C. Zhang \& D. Zou, "Conductive mesh based flexible dye-sensitized solar cells", Applied Physics Letters 90 (2007) 073501.

[18] D. S. Parker, F. Zhang, Y. S. Kim, R. I. Kaiser, A. Landera, V. V. Kislov, A. M. Mebel \& A. Tielens, "Low temperature formation of naphthalene and its role in the synthesis of PAHs (polycyclic aromatic hydrocarbons) in the interstellar medium", Proceedings of the National Academy of Sciences 109 (2012) 53.

[19] H. Ali, R. Seidel, A. Bergmann \& B. Winter, "Electronic structure of aqueous-phase anatase titanium dioxide nanoparticles probed by liquid jet photoelectron spectroscopy", Journal of Materials Chemistry A 7 (2019) 6665-6675.

[20] M. Mohamad, B. U. Haq, R. Ahmed, A. Shaari, N. Ali \& R. Hussain,
"A density functional study of structural, electronic and optical properties of titanium dioxide: Characterization of rutile, anatase and brookite polymorphs", Materials Science in Semiconductor Processing 31 (2015) 405.

[21] H. Xing-Gang, L. An-Dong, H. Mei-Dong, L. Bin \& W. Xiao-Ling, "First-principles band calculations on electronic structures of Ag-doped rutile and anatase $\mathrm{TiO}_{2}$ ", Chinese Physics Letters 26 (2009) 077106.

[22] R. Faccio, L. Fernández-Werner, H. Pardo \& A. W Mombru, "Current trends in materials for dye sensitized solar cells", Recent patents on nanotechnology 5 (2011) 46.

[23] D. Reyes-Coronado, G. Rodríguez-Gattorno, M. Espinosa-Pesqueira, C. Cab, R.d. de Coss \& G. Oskam, "Phase-pure $\mathrm{TiO}_{2}$ nanoparticles: anatase, brookite and rutile", Nanotechnology 19 (2008) 145605.

[24] P. J. Perdew, K. Burke \& M. Ernzerhof, "Generalized gradient approximation made simple", Physical Review Letters18 (1996) 3865.

[25] D. J. Chadi, "Special points for Brillouin-zone integrations", Physical Review B 16 (1977) 1746.

[26] S. S. Alhassan, A. Shuaibu \& M. Y. Onimisi, "Structural and Electronic Properties of Delafossite $C u G a 1 x M n x O 2(X=0.5)$ Nanocomposite: A First Principle Study",Physics Memoir-Journal of Theoretical \& Applied Physics 1 (2019) 106.

[27] H. Dorian AH, M. H. Assadi, S. Li, A. Yu \& C. C. Sorrell, "Ab initio study of phase stability in doped $\mathrm{TiO}_{2}$ ", Computational Mechanics $\mathbf{5 0}$ (2012) 185.

[28] M. Mazmira, B. U. Haq, R. Ahmed, A. Shaari, N. Ali \& R. Hussain, "A density functional study of structural, electronic and optical properties of titanium dioxide: Characterization of rutile, anatase and brookite polymorphs", Materials Science in Semiconductor Processing31 (2015) 405.

[29] S. Raphael, M. Kraft \& O. R. Inderwildi, "Electronic and optical properties of aluminium-doped anatase and rutile $\mathrm{TiO}_{2}$ from ab initio calculations", Physical Review B 81 (2010) 075111.

[30] P. Sandeep, A. Abate, P. Ruckdeschel, B. Roose, Karl C. Gödel, Yana Vaynzof \& Aditya Santhala, "Performance and stability enhancement of dye-sensitized and perovskite solar cells by $\mathrm{Al}$ doping of $\mathrm{TiO}_{2}$ ", Advanced Functional Materials 24 (2014) 6046.

[31] L. Min, J. Zhang \& Y. Zhang, "First-principles calculation of compensated $(2 \mathrm{~N}, \mathrm{~W})$ codoping impacts on band gap engineering in anatase $\mathrm{TiO}_{2}$ ", Chemical Physics Letters 527 (2012) 63.

[32] C. Pawan, P. Basyach \& A. Choudhury, "Structural, optical and photocatalytic properties of $\mathrm{TiO}_{2} / \mathrm{SnO}_{2}$ and $\mathrm{SnO}_{2} / \mathrm{TiO}_{2}$ core-shell nanocomposites: an experimental and DFT investigation", Chemical Physics $\mathbf{4 3 4}$ (2014) 1. 